\title{
Dynamic Modeling \& Stability Analysis of a Generic UAV in Glide Phase
}

\author{
Imran Mir'a $^{\text {, Adnan Maqsood }}{ }^{1}$ and Suhail Akhtar ${ }^{2}$ \\ ${ }^{1}$ Research Center for Modeling and Simulation, National University of Sciences and Technology, H-12 Campus, \\ Islamabad, Pakistan \\ ${ }^{2}$ College of Aeronautical Engineering (CAE), National University of Sciences \& Technology, Risalpur, Pakistan
}

\begin{abstract}
In this paper, we present dynamic modelling and stability analysis of a generic UAV in the glide phase under engine failure condition. When such extreme phenomena occurs, the most desirable requirement is to survive that stage by keeping the vehicle controllable by maintaining its orientation and to glide the vehicle towards the intended direction with maximum extended range. This study investigates the stability aspects of one such aerial vehicle under engine failure condition. In the proposed architecture, a six degree of freedom vehicle dynamic simulation model is implemented through a set of coupled non-linear differential equations. The aerodynamic forces and moments encountered by the UAV during various phases of the flight are ascertained through empirical / non-empirical techniques. Nonlinear constrained optimization technique is employed to evaluate the steady state values of the optimized trajectory for the complete flight regime. Results from dynamical systems theory are applied to investigate local stability characteristics of UAV around the steady state. Complete set of dynamic modes of UAV throughout the glide phase are evaluated and mode content in each of the motion variable is determined using modal decomposition technique. The dynamic characteristics of the open-loop configuration are assessed to generate adequate benchmark performance for closed-loop controller design
\end{abstract}

\section{Introduction}

Unmanned Air Vehicles (UAV) have poor safety records than manned counterparts [1, 2]. By bringing human out of the cockpit, both mission and cost effectiveness are enhanced by multiple folds. Medium Altitude Long Endurance (MALE) [3] or High Altitude Long Endurance (HALE) [4] configurations are worthy of performing Dull, Dangerous and Dirty (DDD) missions. However, since the safety factor during design considerations of UAVs is relatively low, therefore, system and subsystem level failures are a common occurrence [5]. This research deals with one undesirable phenomenon of engine failure during flight. Generally, UAVs fly with one engine on-board and typically are equipped with pusher type propeller. The engine failures can be caused by mechanical or electrical malfunctioning or as a result of enemy fire. Under such scenarios, successful retrieval of the platform in integrated state back at the operating base becomes of vital importance. In this paper, dynamic modeling and stability analysis is carried out for one such generic Unmanned Air Vehicle with two all moving control surfaces, which can stabilize and accurately navigate to a reasonably

${ }^{\text {a } C o r r e s p o n d i n g ~ a u t h o r ~: ~ i m r a n m i r 56 @ y a h o o m a i l . c o m ~}$ 
distant base station in case of engine failure. In this proposed architecture, a 6-DOF vehicle modeling is performed through a set of highly coupled non-linear differential equations [6]. In order to evaluate aerodynamic forces and moments during various phases of the flight, empirical and non-empirical techniques such as Computational Fluid Dynamics (CFD) [7], USAF Stability and Control Digital Datcom [8] and so on [9] were employed. Non-linear constrained optimization technique is employed to evaluate the steady state values of the optimized trajectory for the complete flight regime to ensure vehicle movement at an optimum path. Trim point analysis and model linearization was performed in Matlab ${ }^{\circledR}$ environment. Eigen value analysis of the linearized system was than performed to determine the response of the open loop system under stated problem. System stability aspects were than evaluated to determine the possibility of designing a suitable control architecture which can keep the system stabilize and ensure that the UAV follows the optimized trajectory under engine failure condition.

\section{UAV Flight Dynamics Model}

Vehicle simulations are commonly designed to work with three-degree of freedom (3 DOF) [10, 11] or six-degree of freedom (6 DOF) [12] equations of motion. Each system has its own pros and cons, which make them more suitable for specific applications. In this study, 6-DOF model because of its capability to cater for both the translational and rotational dynamics is utilized to model UAV dynamics.

\subsection{UAV Equation of Motion}

The rigid aircraft motion equations are presented in the form of system of first order nonlinear differential equations describing translational/ angular accelerations and kinematics of angular motion The governing equations (refer equation(1)-(12)) based upon two valid assumptions of flat and nonrotating Earth, are given below:-

$$
\begin{aligned}
& \left.\dot{U}=R V-Q W-g_{D} \sin \theta+\left(X_{A}+X_{T}\right) / m\right) \\
& \dot{V}=-R U+P W+g_{D} \sin \phi \cos \theta+\left(Y_{A}+Y_{T}\right) / m \\
& \dot{W}=Q U-P V+g_{D} \cos \phi \cos \theta+\left(Z_{A}+Z_{T}\right) / m \\
& \dot{\phi}=P+\tan \theta(Q \sin \phi+R \cos \phi) \\
& \dot{\theta}=Q \cos \phi-R \sin \phi \\
& \dot{\psi}=(Q \sin \phi+R \cos \phi) / \cos \theta \\
& \dot{\Gamma}=J_{x z}\left[J_{x}-J_{y}+J_{z}\right] P Q-\left[J_{z}\left(J_{z}-J_{y}\right)+J_{x z}^{2}\right] Q R+J_{z} l+J_{x z}{ }^{n} \\
& J_{y} \dot{Q}^{2}\left(J_{z}-J_{x}\right) P R-J_{x z}\left(P^{2}-R^{2}\right)+m \\
& \dot{\Gamma}=\left[\left(J_{x}-J_{y}\right) J_{x}+J_{x z}^{2}\right] P Q-J_{x z}\left[J_{x}-J_{y}+J_{z}\right] Q R+J_{x z} l+J_{x}{ }^{n} \\
& \dot{x}=U c \theta c \psi++V(-c \phi s \psi+s \phi s \theta c \psi)+W(s \phi s \psi+c \phi s \theta c \psi) \\
& \dot{y}=U c \theta s \psi+V(c \phi c \psi+s \phi s \theta s \psi)+W(-s \phi c \psi+c \phi s \theta s \psi) \\
& \dot{h}=U s \theta-V s \phi c \theta-W c \phi c \theta
\end{aligned}
$$


where

$$
\begin{aligned}
& U=V_{T} \cos \alpha \cos \beta, V=V_{T} \sin \beta, W=V_{T} \sin \alpha \cos \beta \\
& X_{A}=\bar{q} S C_{x}, \quad Y_{A}=\bar{q} S C_{y}, \quad Z_{A}=\bar{q} S C_{z}, \Gamma=J_{x} J_{z}-J_{x z}^{2}
\end{aligned}
$$

also $\phi, \theta, \psi, \mathrm{P}, \mathrm{Q}, \mathrm{R}$ are the roll, pitch yaw angles and rates respectively, $\mathrm{U}, \mathrm{V}, \mathrm{W}$ and $J_{\otimes}$ are the velocity components and moments along the three axis, $\gamma$ is the flight path angle, $\mathrm{X}_{\mathrm{A}}, \mathrm{X}_{\mathrm{T}}$ are the axial and tangential forces

\subsection{Aerodynamic Parameters Estimation}

Multi-dimensional look-up tables based high fidelity aerodynamic model is developed from experimental data obtained by employing various empirical and non-empirical techniques. Broadly each aerodynamic coefficient in aircraft body axes system is expressed as a summation of aerodynamic effects due to static affects and dynamic affects (refer equation (13)).

$$
C_{i}=C_{i, \text { static }}(\alpha, \beta, \delta, M)+C_{i, \text { dynamic }}(\dot{\alpha}, \dot{\beta}, p, q, r)
$$

where

$$
C_{i}=C_{L}, C_{D}, C_{Y}, C_{l}, C_{m}, C_{n}
$$

Different methods were subsequently adopted to ascertain the static and dynamic components of these aerodynamic coefficients. Evaluation of static (basic) coefficient data (refer equation (14)) was performed employing Computational Fluid Dynamics (CFD) [7, 13] technique. All the parameters were obtained as a function of control effort, angle of attack $(\alpha)$, side slip $(\beta)$ and mach number (M).

$$
C_{i, \text { static }}=C_{D b}, C_{L b}, C_{Y b}, C_{l b}, C_{m b}, C_{n b}
$$

Dynamic coefficient data (refer equation (15)) consisting of damping/rate derivatives and acceleration derivatives were calculated utilizing 'USAF stability and Control DATCOM' [8] and other empirical techniques [9].

$$
C_{i, \text { dynamic }}=\text { dampingderivatives }+ \text { Accelerationderivatives }
$$

where the damping and acceleration derivatives are defined in equation (16) and equation (17) respectively

$$
\begin{aligned}
& \text { dampingderivatives }=\left(C_{Y r}, C_{l r}, C_{n r}\right)+\left(C_{Y p}, C_{l p}, C_{n p}\right)+\left(C_{Y r}, C_{l r}, C_{n r}\right) \\
& \text { accelerationderivatives }=\left(C_{L \dot{\alpha}}, C_{D \dot{\alpha}}, C_{m \dot{\alpha}}\right)+\left(C_{Y \dot{\beta}}, C_{l \dot{\beta}}, C_{n \dot{\beta}}\right)
\end{aligned}
$$

Through this accurate estimation of the aerodynamic affects (refer equation (18)) were ascertained for the complete flight regime. 


$$
\begin{aligned}
& C_{Y}=C_{Y b}+C_{Y \dot{\beta}} * \dot{\beta}^{*}\left(b / 2 V_{T}\right)+C_{Y p} * p^{*}\left(b / 2 V_{T}\right)+C_{Y r} * r^{*}\left(b / 2 V_{T}\right), C_{l}=C_{l b}+C_{l \dot{\beta}} * \dot{\beta}^{*}\left(b / 2 V_{T}\right)+C_{l p} * p^{*}\left(b / 2 V_{T}\right)+C_{l r} * r^{*}\left(b / 2 V_{T}\right) \\
& C_{L}=C_{L b}+C_{L \dot{\alpha}} * \dot{\alpha} *\left(\bar{c} / 2 V_{T}\right)+C_{L q} * q^{*}\left(\bar{c} / 2 V_{T}\right), \quad C_{D}=C_{D b}+C_{D \dot{\alpha}} * \dot{\alpha} *\left(\bar{c} / 2 V_{T}\right)+C_{D q} * q^{*}\left(\bar{c} / 2 V_{T}\right), \\
& C_{m}=C_{m b}+C_{m \dot{\alpha}} \cdot \dot{\alpha} \alpha^{*}\left(\bar{c} / 2 V_{T}\right)+C_{m q} * q^{*}\left(\bar{c} / 2 V_{T}\right), C_{n}=C_{n b}+C_{n \dot{\beta}}{ }^{*} \dot{\beta}^{*}\left(b / 2 V_{T}\right)+C_{n p} * p^{*}\left(b / 2 V_{T}\right)+C_{n r} * r^{*}\left(b / 2 V_{T}\right)
\end{aligned}
$$

\section{Trajectory Optimization}

\subsection{Generation of Optimized Velocity Profile}

Generation of optimized velocity profile (ascertaining velocity, dynamic pressure and Mach no for the complete flight regime) that yields the greatest performance plays a substantial role in the effective design of flight vehicles. This is important since the use of ad-hoc profile or control policies to evaluate competing configurations may inappropriately penalize the performance of one configuration over another. Thus, to guarantee optimized trajectory, it is important to optimize the velocity profile and control policy for each configuration early in the design process. Subsequently, two different types of optimized velocity profiles were created to acquire ranges of $100 \mathrm{~km}$ and $120 \mathrm{~km}$ respectively once vehicle engine failure condition occurs (refer 'figure 1')
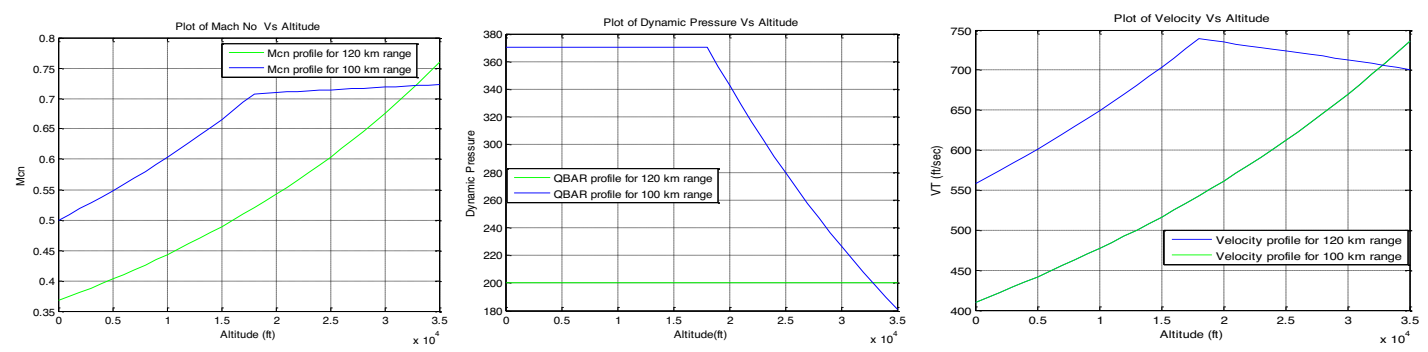

Figure 1. Generation of optimized velocity

In regards to the velocity profile for $328000 \mathrm{ft}(100 \mathrm{~km})$ range, dynamic pressure of 370.5 is maintained for the altitude ranging from 0 to $18000 \mathrm{ft}$. Using the standard atmospheric model, Mach number (M) and Velocity (VT) to have the desired altitude and dynamic pressure are subsequently ascertained. For high altitudes (19000-35000ft) dynamic pressure is slowly varied to maintain desired mach number (refer Table 1). Similarly velocity profile for $120 \mathrm{~km}$ range was generated by maintaining a constant dynamic pressure of 200 at all the altitudes $(0-35000 \mathrm{ft})$. Using the standard atmospheric model, corresponding Mach number (M) and Velocity (VT) to have the desired altitude and dynamic pressure were calculated for all the individual 36 flight conditions $(0-35000 \mathrm{ft}$ with an increment of 1000). 
Table 1. Velocity profile for achieving $100 \mathrm{~km}$ range

\begin{tabular}{|l|l|l|l|l|l|l|l|l|l|}
\hline No & $\begin{array}{l}\text { Altitude } \\
\text { (ft) }\end{array}$ & $\begin{array}{l}\text { Velocity } \\
(\mathbf{f t} / \mathbf{s e c})\end{array}$ & Mach No & $\begin{array}{l}\text { Dynamic } \\
\text { Pressure }\end{array}$ & No & $\begin{array}{l}\text { Altitude } \\
\text { (ft) }\end{array}$ & $\begin{array}{l}\text { Velocity } \\
\text { (ft/sec) }\end{array}$ & $\begin{array}{l}\text { Mach } \\
\text { No }\end{array}$ & $\begin{array}{l}\text { Dynamic } \\
\text { Pressure }\end{array}$ \\
\hline 1 & 0 & 558.334 & 0.500 & 370.5 & 19 & 18000 & 738.79 & 0.707 & 370.5 \\
\hline 2 & 1000 & 566.54 & 0.50 & 370.5 & 20 & 19000 & 736.74 & 0.708 & 356.32 \\
\hline 3 & 2000 & 574.94 & 0.51 & 370.5 & 21 & 20000 & 734.67 & 0.709 & 342.57 \\
\hline 4 & 3000 & 583.521 & 0.52 & 370.5 & 22 & 21000 & 732.56 & 0.710 & 329.24 \\
\hline 5 & 4000 & 592.292 & 0.53 & 370.5 & 23 & 22000 & 730.47 & 0.711 & 316.31 \\
\hline 6 & 5000 & 601.260 & 0.54 & 370.5 & 24 & 23000 & 728.35 & 0.712 & 303.78 \\
\hline 7 & 6000 & 610.431 & 0.55 & 370.5 & 25 & 24000 & 726.26 & 0.713 & 291.65 \\
\hline 8 & 7000 & 619.811 & 0.57 & 370.5 & 26 & 25000 & 724.03 & 0.714 & 279.89 \\
\hline 9 & 8000 & 629.406 & 0.58 & 370.5 & 27 & 26000 & 721.84 & 0.715 & 268.51 \\
\hline 10 & 9000 & 639.223 & 0.59 & 370.5 & 28 & 27000 & 719.63 & 0.715 & 257.49 \\
\hline 11 & 10000 & 649.269 & 0.60 & 370.5 & 29 & 28000 & 717.40 & 0.716 & 246.83 \\
\hline 12 & 11000 & 659.55 & 0.61 & 370.5 & 30 & 29000 & 715.14 & 0.717 & 236.51 \\
\hline 13 & 12000 & 670.076 & 0.627 & 370.5 & 31 & 30000 & 712.8703 & 0.718 & 226.541 \\
\hline 14 & 13000 & 680.852 & 0.639 & 370.5 & 32 & 31000 & 710.5723 & 0.719 & 216.8965 \\
\hline 15 & 14000 & 691.88 & 0.652 & 370.5 & 33 & 32000 & 708.252 & 0.720 & 207.5758 \\
\hline 16 & 15000 & 703.190 & 0.665 & 370.5 & 34 & 33000 & 705.9092 & 0.721 & 198.571 \\
\hline 17 & 16000 & 714.769 & 0.679 & 370.5 & 35 & 34000 & 703.5435 & 0.722 & 189.8744 \\
\hline 18 & 17000 & 726.632 & 0.693 & 370.5 & 36 & 35000 & 699.9891 & 0.721 & 180.8756 \\
\hline
\end{tabular}

\subsection{Trajectory Optimization}

In order to ascertain steady states for the optimized trajectory, the problem was formulated as nonlinear dynamic optimization problem, with an objective to determine, in open loop control, a set of time dependent decision variables that optimizes a specified performance index subject to certain constrains [14]. Matlab optimization toolbox was used to solve the problem utilizing fminsearch solver which is based upon 'Nelder-Mead simplex direct search' algorithm [15]. Steady state for the optimized trajectory were subsequently obtained for a coordinated turn flight as per the following constraints (refer equation (19)-(24))

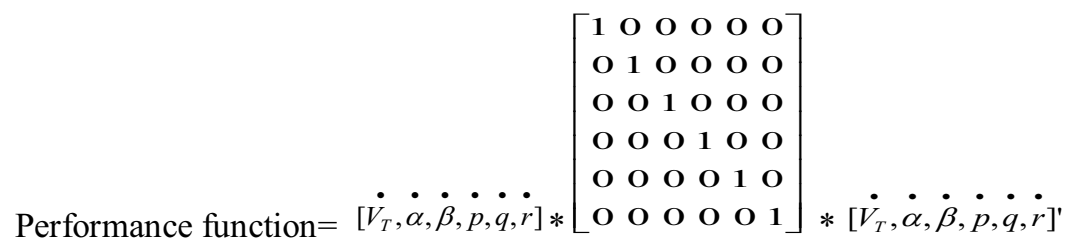

Fixed States $=\left[V_{T} ; \boldsymbol{h} ; \gamma ; \boldsymbol{\psi} ; \dot{\phi} ; \dot{\theta} ; \dot{\boldsymbol{\psi}}\right]$

Variable States $=[\alpha ; \beta ; L C F ; R C F]$

Constrained States $=[\phi ; \theta ; p ; q ; r]$

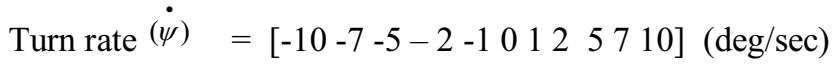

Altitude $=[1000: 1000: 35000])$

Where $[\mathrm{LCF}, \mathrm{RCF}]$ is the control vector for left and right control fins and all other variables already defined. 
This makes a total of $35 \times 11$ flight conditions, about which the trim points (for both states and controls) were ascertained for the generation of optimized vehicle trajectory. This enhanced flight envelope frame work was developed to ensure controlled vehicle movement under a wide range of operating conditions.

\section{Open Loop Analysis}

\subsection{Model Linearization \& Eigen Value Analysis}

In order to linearize the non-linear system about the steady states trim points, simulink model was developed in matlab environment [16], in which the 6 DOF non-linear model along with all dependencies were incorporated. Matlab linearization tool linmod was used for model linearization, as a result of which, linearized state space representation of the model about the trim point was obtained for the optimized trajectory. Open loop analysis of the linearized system, revealed that system is unstable as it contained eigen values in open right half plane at various flight conditions.

As an example linearized system response at two different flight conditions corresponding to altitude $10,000 \mathrm{ft}, \dot{\psi}=0 \mathrm{deg}$ and altitude $30,000 \mathrm{ft}, \dot{\psi}=0 \mathrm{deg}$ is depicted at 'figure 2', which is clearly unstable as it contains eigen value in the open right half plane. The open loop unstable eigen values at various flight conditions was the cause of vehicle instability and movement in an uncontrolled manner under engine failure condition. System uncontrolled behavior was subsequently analyzed to ascertain system state response and to determine the feasibility of design of a control architecture which can stabilize the system and ensure system stability under such stated problem.
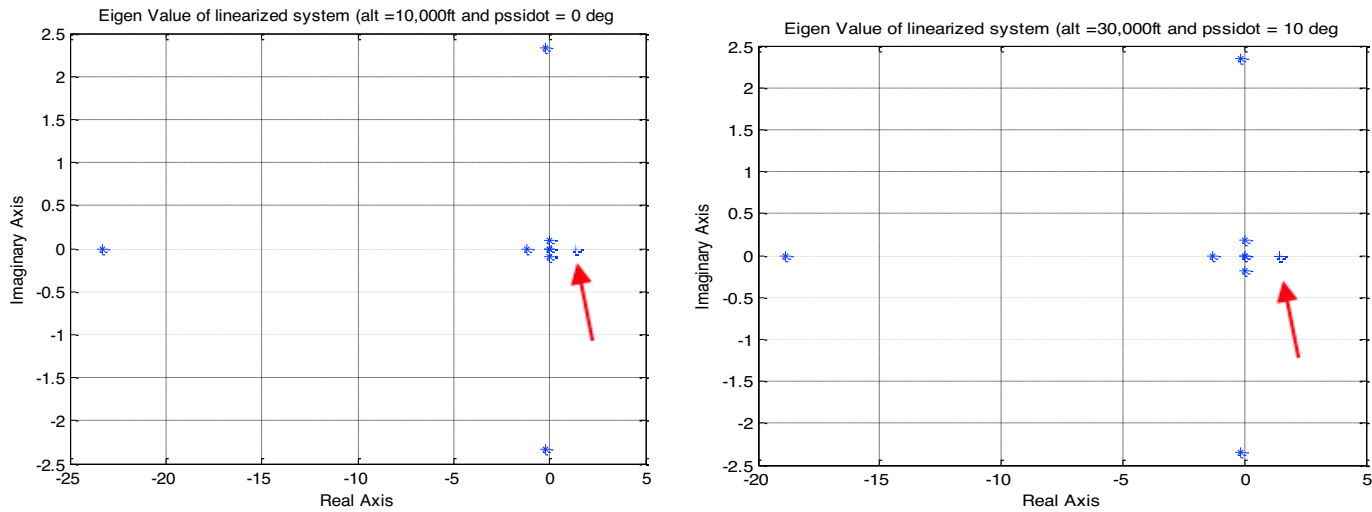

Figure 2. Eigen value analysis

\subsection{Open Loop System Response}

In order to analyze system response under engine failure condition, a simulink model was developed in the matlab environment. In this, the vehicle once subjected to the stated problem, showed a complete unstable response. The UAV instead of following the optimized trajectory refer 'figure 3 (a)', exhibited an unstable response with a glide range of just $15 \mathrm{Km}$ (refer 'figure 3 (b)'). This problem of low range was encountered as the vehicle after a short time became totally unstable and exhibited a steep vertical decent instead of following the optimized trajectory. 

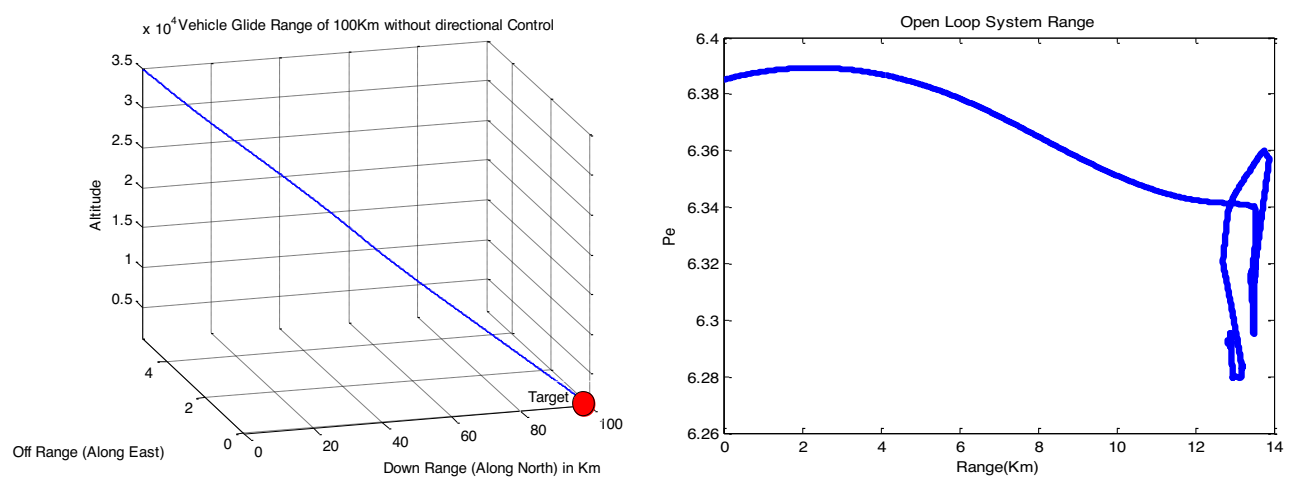

Figure 3. (a) Desired response (b) Actual system response

Apart from low range, system longitudinal and lateral dynamics also exhibited unstable responses (refer 'figure 4').
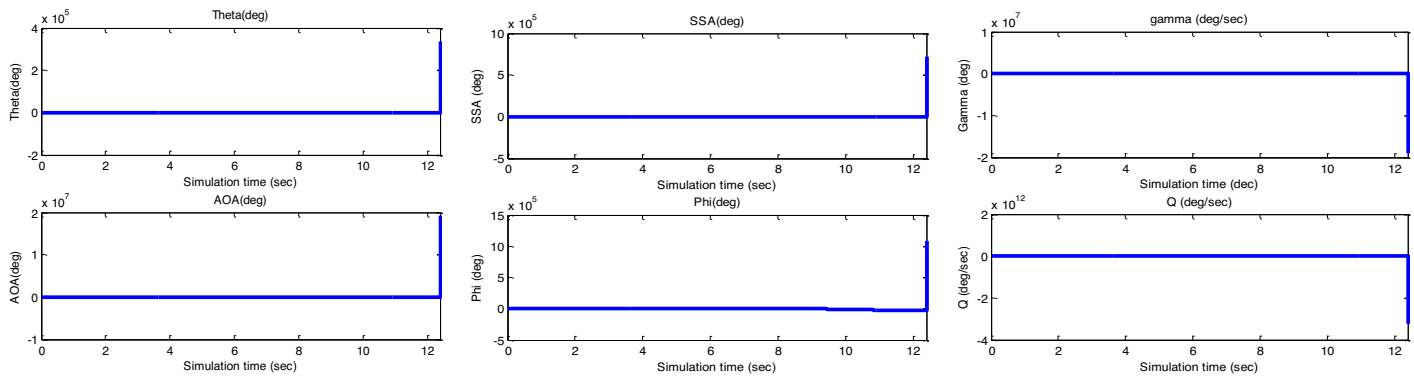

Figure 4. Open loop system unstable response

\subsection{Stability Analysis}

In order to keep the linearized system stable about the operating points and to make the system follow desired trajectory, requirement existed for the presence of a closed loop system with dedicated control architecture. Linearized system when evaluated for controllability and observability was found to be both controllable and observable. This ensured that control architecture can always be designed for close loop system stability and with the incorporation of appropriate stability controller, system states can be forced to follow the optimized trajectory. To design a closed loop system which can follow the optimized trajectory, response of various types of controllers such as full state feedback LQR controller [17], PID controller [18] (both SISO [19] and MIMO [20]) can be investigated. A promising choice in this regard could be the utilization of robust state feedback LQR controller in case the states are measurable or else an associated Kalman filter [21] can be designed for the state estimation. Implementation of such robust architecture will not only ensure close loop system stability but will also dramatically reduce the sensitivity of the system to modeling uncertainties and provides disturbance rejection to those quantities that detract from the desired aircraft motion. Apart from this, much enhanced vehicle glide phase range will also be achieved in comparison to the open loop range of just $15 \mathrm{~km}$. This range enhancement is a consequence of the system states forced to follow the optimized trajectory. In case controller is designed utilizing 6 DOF equations of motion, problem of system tracking the intended direction might be experienced. This is so because the estimates for directional orientation provided by navigational equation (refer equation (10-12)) are not used by other state equations. Due this reason, heading angle $(\psi)$ is not controlled as per the actual direction of flight. Accurate navigation to the target, therefore requires incorporation of an additional tracking 
controller which utilizes the orientation information provided by navigational equation to compute the desired heading and then compares it with current heading to compute the difference in heading for necessary compensation. This computed value may be then subsequently utilized for steering the aircraft in the desired direction. With the incorporation of suitable close loop stabilization controller and a tracking controller, control system architecture can be developed to ensure UAV survivability under stated problem, and that can keep the vehicle stable and glide towards intended direction.

\section{Conclusion}

In this study, dynamic modelling and stability analysis of UAV was performed under engine failure condition. Vehicle dynamic simulation model was implemented through a set of highly coupled nonlinear 6 DOF differential equations. Established empirical/non-empirical techniques were employed to accurately determine, the aerodynamic affects during various phases of the flight. Steady state analysis of the linearized system revealed various unstable modes that caused vehicle instability and short range of just few $\mathrm{km}$. System was however ascertained to be fully controller and observable that guaranteed design of a close loop system for system stability. Vehicle response under stated problem was analyzed and a framework was presented to ensure vehicle survivability. Simulations results depicted considerable enhancement in the UAV range to $100 \mathrm{~km}$ from free fall unguided range of just $15 \mathrm{~km}$. The potential benefit provided by this study remains two folds in which not only considerable range enhancement is achieved to attain high percentage of vehicle survivability but the intrinsic simplicity of the optimization algorithm guarantee its on board implementation. At the end a suitable control architecture comprising of stabilization and tracking controllers was also briefly discussed (scope of next work) that could provide steering commands to follow the optimized trajectory.

\section{References}

1. Dalamagkidis, K., K. Valavanis, and L. Piegl, On unmanned aircraft systems issues, challenges and operational restrictions preventing integration into the National Airspace System. Progress in Aerospace Sciences, 2008. 44(7): p. 503-519.

2. Wild, G., J. Murray, and G. Baxter, Exploring drone accidents and incidents to help prevent potential air disasters. Aerospace, 2016. 3(3): p. 1-11.

3. Scarselli, G., L. Lecce, and F. Nicolosi, DEVELOPMENT OF A MALE TURBO-PROP

UNMANNED AERIAL VEHICLE FOR CIVIL APPLICATION. Aerotecnica Missili \& Spazio, 2016.

86(1): p. 22-35.

4. Cestino, E., Design of solar high altitude long endurance aircraft for multi payload \& operations. Aerospace science and technology, 2006. 10(6): p. 541-550.

5. Nickol, C.L., et al., High altitude long endurance air vehicle analysis of alternatives and technology requirements development. AIAA Paper, 2007. 1050: p. 2007.

6. Roaskam, J., Airplane Flight Dynamics and Automatic Flight Controls. Vol. Part1. 2001:

DARcoperation, USA.

7. Petterson, K., CFD analysis of the low-speed aerodynamic characteristics of a UCAV. AIAA Paper, 2006. 1259: p. 2006.

8. USAF Stability and Control Datcom. Vol. 1. 1999: McDonnell Douglas Astronautics Company,

St. Louis Division, St Louis, Missouri 63166.

9. Roskam, D.J., Airplane Design Vol 1-7. Roskam Aviation and Engineering Corporation. 1985

10. Tischler, M.B. and R.K. Remple, Aircraft and rotorcraft system identification. AIAA education series, 2006.

11. Wickenheiser, A.M. and E. Garcia, Optimization of perching maneuvers through vehicle morphing. Journal of Guidance, Control, and Dynamics, 2008. 31(4): p. 815-823.

12. Jung, D. and P. Tsiotras, Modeling and hardware-in-the-loop simulation for a small unmanned aerial vehicle. AIAA Infotech at Aerospace, AIAA, 2007: p. 07-2763.

13. Buning, P.G., R.J. Gomez, and W.I. Scallion, CFD approaches for simulation of wing-body stage 
separation. AIAA Paper, 2004. 4838: p. 2004.

14. Maqsood, A. and T. Hiong Go, Longitudinal flight dynamic analysis of an agile UAV. Aircraft Engineering and Aerospace Technology, 2010. 82(5): p. 288-295.

15. Lagarias, J.C., et al., Convergence properties of the Nelder--Mead simplex method in low dimensions. SIAM Journal on optimization, 1998. 9(1): p. 112-147.

16. Li, H., X.-G. Jing, and L.-M. Xu, The Simulation of 6-DOF Motion of Launch Vehicle Based on Matlab/Simulink [J]. Journal of Astronautics, 2005. 5: p. 016.

17. Hespanha, J.P., LQG/LQR controller design. Undergraduate Lecture Notes, University of California, Santa Barbara, California, USA, 2007.

18. Rivera, D.E., M. Morari, and S. Skogestad, Internal model control: PID controller design. Industrial \& engineering chemistry process design and development, 1986. 25(1): p. 252-265.

19. Ramasamy, M. and S. Sundaramoorthy, PID controller tuning for desired closed-loop responses for SISO systems using impulse response. Computers \& Chemical Engineering, 2008. 32(8): p. 17731788.

20. Vilanova, R. and A. Visioli, PID control in the third millennium. 2012: Springer.

21. Hajiyev, C. and S.Y. Vural, LQR controller with Kalman estimator applied to UAV longitudinal dynamics. Positioning, 2013. 4(01): p. 36. 